\title{
A Transistor-Like Device for Light
}

\section{A new metamaterial device can amplify and manipulate electromagnetic waves, a capability necessary for advancing photonics platforms.}

\author{
By Sophia Chen
}

$\prod$

he transistor is an electronic device that is used to control the flow of current in a circuit. For example, the metal-oxide-semiconductor field-effect transistor (MOSFET), one of the most popular types of transistor, can be used to regulate current by applying an electric field to the MOSFET's gate, changing the device's conductivity. Researchers think that photonics platforms-emerging technologies that perform computations using light-could also benefit from transistor-like devices, but ones that control the propagation of electromagnetic waves. Now, Sylvain Lannebère of the University of Coimbra in Portugal and colleagues predict a transistor-like response to light from a 3D nonlinear metamaterial subject to a static electric field [1]. These devices could be used for fast information transmission and computation via photons.

The team presented a concept for a nonlinear metamaterial whose permittivity changes with an applied static electric field. The nonlinear response is such that an electric field applied along one axis can change the material's permittivity along

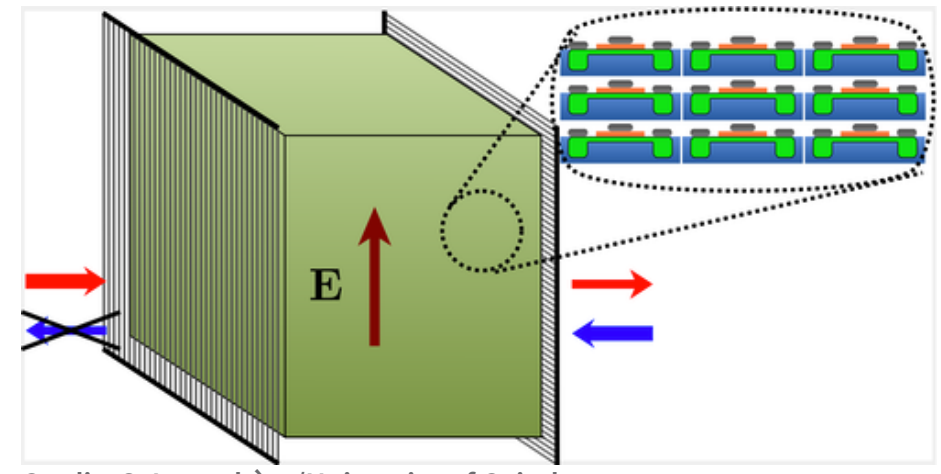

Credit: S. Lannebère/University of Coimbra another axis. The team predicts that, when this electric field is on, the interaction between the metamaterial and an incoming light wave can either increase or decrease the amplitude of that wave, depending only on the light wave's polarization. This amplitude change means the metamaterial could be engineered to either amplify or attenuate an incoming light signal.

The team devised a device consisting of the metamaterial layer sandwiched between two orthogonal linear polarizers. They showed that the device can act as an isolator, transmitting electromagnetic signals in only one direction. Lannebère and his colleagues are currently investigating platforms to physically realize their device, with one possibility being to use arrays of transistors to make the metamaterial slab.

Sophia Chen is a freelance science writer based in Columbus, Ohio.

\section{REFERENCES}

1. S. Lannebère et al., "Nonreciprocal and non-Hermitian material response inspired by semiconductor transistors," Phys. Rev.

Lett. 128, 013902 (2022). 